\title{
Trace and long-delay fear conditioning in the developing rat
}

\author{
ROBERT C. BARNET and PAMELA S. HUNT \\ College of William \& Mary, Williamsburg, Virginia
}

\begin{abstract}
In two experiments with rats, we examined the developmental emergence of conditioned freezing following trace and short-delay conditioning and also included a long-delay comparison group. In the short-delay and trace groups, a 10-sec conditioned stimulus (CS) was paired with shock; for the trace rats, a 10-sec trace interval followed CS termination. The long-delay groups received a 20 -sec CS paired with shock, to equate for the longer interstimulus interval (ISI) in the trace group. Trace conditioning emerged later in development than did short-delay conditioning (see Moye \& Rudy, 1987). Importantly, long-delay conditioning emerged in parallel with trace conditioning, at a similar time, and with similar strength. These findings suggest a role for the longer ISI, as opposed to the unfilled gap per se, in the late emergence of trace conditioning. The role of the hippocampus in trace conditioning and the possibility that young rats encode the temporal relationship between CSs and unconditioned stimuli are also considered.
\end{abstract}

In Pavlovian fear conditioning, a conditioned stimulus (CS), such as a tone or light, is paired with an unconditioned stimulus (US), such as shock. In delay conditioning, the CS and the US typically overlap and coterminate, ending the trial. CS-US pairings in the delay procedure result in rapid acquisition of fear to the $\mathrm{CS}$, as evidenced by CS-elicited defensive reactions such as freezing, changes in autonomic function, and potentiation of the acoustic startle reflex (e.g., Davis, 1992; Hunt \& Campbell, 1997; LeDoux, 2000). In the procedure referred to as trace conditioning, the CS is presented and is later followed, after a stimulus-free period (trace interval), by presentation of the US. Trace conditioning typically results in comparably weaker conditioning, and responding to the CS declines as the length of the trace interval increases (Ellison, 1964; Kamin, 1954).

Moye and Rudy (1987) have presented an extensive analysis of the development of delay and trace fear conditioning in the rat. There was a clear age-related increase in the animals' abilities to learn as the trace interval was lengthened. Specifically, Moye and Rudy reported that animals as young as 15 days of age could exhibit conditioned suppression to a tone that was paired with shock in a 0 -sec trace (i.e., delay) procedure, but it was not until 17 days of age that learning was evident with a 10 -sec trace interval. Moreover, the animals were able to span a 30 -sec trace interval at 21 days, but not at younger ages. This same developmental trend was observed when the

This research was supported by National Institute of Alcohol Abuse and Alcoholism Grant AA12466 to P.S.H. The authors thank Brandi Rima, Deborah Murdoch, and Sara Meier for their assistance with data collection. Correspondence concerning this article should be addressed to R. C. Barnet, Department of Psychology, College of William \& Mary, P.O. Box 8795, Williamsburg, VA 23187-8795 (e-mail: rcbarn@ wm.edu).
CS was a visual stimulus. Conditioned suppression to a light paired with shock in the $0-, 10-$, and 30 -sec trace groups was evident at 17,25 , and 30 days of age, respectively. These data indicate that the ability to acquire fear in a trace conditioning paradigm improves during the first several weeks of life and suggest that short-term memory abilities progressively increase during this time. Moreover, the animals were able to learn about an auditory cue at a younger age than they were able to learn about a visual cue. This sequential development of learning across different sensory systems is a robust finding in the developmental literature (e.g., Alberts, 1984; Campbell \& Ampuero, 1985; Hunt \& Campbell, 1997).

A potential problem in many studies of trace conditioning has been the comparison delay group against which trace conditioning has been assessed. The trace conditioning procedure differs from delay conditioning in two important respects. First, the trace procedure includes a stimulus-free period between CS offset and US onset. The difficulty with acquisition of trace conditioning and lower asymptotic performance has usually been attributed to the presence of this trace interval. However, a second major difference between these two paradigms is the interstimulus interval (ISI) - that is, the time from CS onset to US onset. If CS duration is maintained across the delay and trace preparations, the ISI is necessarily longer in the trace procedure. Therefore, inclusion of a comparison group that equates ISI across standard delay and trace conditions is needed in order to appropriately examine the underlying basis of the trace conditioning deficit. A long-delay procedure controls for the increase in ISI, while maintaining the CS-US temporal relationship of the standard short-delay group.

In a study in which the ontogeny of trace eyeblink conditioning in the rat was examined, Ivkovich, Paczkowski, and Stanton (2000) reported that trace conditioning was 
first evident at older ages than was standard short-delay conditioning and that long-delay conditioning emerged in parallel with trace conditioning. These results suggest that, at least in young animals, the ISI plays a critical role in the acquisition of trace conditioned responding. Thus, the deficit in trace eyeblink conditioning seems not to be due entirely to the development of short-term memory processes but, rather, results, at least in part, from the young animal's inability to form associations across long ISIs.

The contribution of a longer ISI, versus the presence of a trace interval, to the late development of trace fear conditioning, however, remains in question, since Moye and Rudy (1987) did not include a long-delay comparison group. Indeed, fear conditioning exhibits a number of characteristics different from those of eyeblink conditioning, including the fact that fear conditioning is acquired at a much younger age than is eyeblink conditioning (Hunt \& Campbell, 1997; Stanton, 2000). The present experiments were intended to further examine trace fear conditioning during development by including long-delay control groups. The experiments, therefore, permit assessment of whether the trace conditioning deficit in a fear paradigm is a function of the typically longer ISI, the presence of the trace interval itself, or a combination of both. If the trace conditioning deficit is due to the trace interval per se, one would anticipate trace conditioning to be inferior to longdelay conditioning. If responding in trace and long-delay groups is equivalent and inferior to that in a short-delay condition, the ISI would be implicated as a primary source of the learning deficit normally observed after trace conditioning. The empirically motivated question in the present experiments was whether long-delay and trace fear conditioning would exhibit a parallel emergence during development, as has been reported for eyeblink conditioning (Ivkovich et al., 2000).

\section{EXPERIMENT 1}

The goal of this experiment was to compare the development of auditory trace and delay fear conditioning in preweanling and juvenile rats, equating for differences in ISI. To accomplish this, two different CS durations were used for delay conditioning. For one group (short delay), the CS duration was equal to that used for the trace group, and for the other group (long delay), the CS duration was longer, allowing the ISI to be matched to that of the trace group. In the common delay conditioning procedure, US onset occurs prior to expiry of the CS, and the CS and the US coterminate. With this procedure, the US always occurs in the presence of the CS. For an otherwise comparable trace procedure, however, the US always occurs in the absence of the CS at the end of the trace interval. Delay and trace conditions would be more equivalent if, in the delay procedure, US onset occurred at expiry of the CS (see Moye \& Rudy, 1987). The present experiment used this type of delay procedure so that the US would always occur in the absence of the CS for all the experi- mental comparisons groups (short delay, long delay, and trace). Finally, a group that was given explicitly unpaired presentations of the CS and the US was included in order to control for stimulus exposure and pseudoconditioning, and for this group as well, the US occurred in the absence of the CS during training.

\section{Method}

Subjects. The subjects in this experiment were 147 male and female rats representing 18 litters. On the day of training, the subjects were $14,16,18$, or 25 days of age. The subjects were offspring of Sprague Dawley derived rats, born and reared in the Psychology Department at the College of William \& Mary. Breeders were obtained from Charles River Laboratories (Wilmington, MA). Male and female breeders were housed together in $50.8 \times 40.6 \times 21.6 \mathrm{~cm}$ polycarbonate cages with wire lids and pine chip bedding. Food (DietLab Formula 5008) and water were available ad lib. The cages were checked daily for pups, and the day of birth was designated as postnatal day (PD) 0 . Litters were culled to $8-10$ pups on PD 2. The vivarium was maintained on a 14:10-h light:dark cycle, with light onset at $0600 \mathrm{~h}$, and all training and testing procedures occurred during the light portion of the cycle. No more than 2 animals per litter ( 1 male and 1 female) were assigned to each conditioning treatment, and each litter contributed subjects to one age group. All the procedures were approved by the Institutional Animal Care and Use Committee at the College of William \& Mary.

Apparatus. Fear conditioning and later behavioral testing were conducted in two different contexts. Fear conditioning occurred in two identical modified Skinner boxes, each measuring $38.0 \times$ $26.0 \times 22.0 \mathrm{~cm}$. The two shorter walls were made of aluminum, and the two longer walls and top were made of Plexiglas. The floor was constructed of 5-mm stainless steel bars spaced $1.5 \mathrm{~cm}$ apart (center to center). The grid floor was connected to a custom-made constant current shock generator that delivered the 0.5 - $\mathrm{mA} 1$-sec shock US. Each chamber was located in a custom-built sound-attenuating shell measuring $67.0 \times 71.5 \times 71.0 \mathrm{~cm}$. A $4-\mathrm{W}$ red bulb was mounted on an inner wall of the sound-attenuating shell to provide constant lowlevel illumination. The auditory CS was an $80-\mathrm{dB}$ (C scale, Simpson Electronics Model 886) $1600-\mathrm{Hz}$ pure tone that pulsed at a rate of $2 / \mathrm{sec}$ (250-msec rise/fall time) presented through a 4-in. speaker mounted in the center of the ceiling of the conditioning chamber. All stimulus presentations were controlled by a PC computer that interfaced Coulbourn Instruments (Allentown, PA) software and hardware.

Testing occurred in a $29.0 \times 21.5 \times 46.5 \mathrm{~cm}$ clear Plexiglas chamber with an open top and bottom. The chamber rested on a Plexiglas floor covered with brown paper. To facilitate transmission of the auditory CS, the lower $11 \mathrm{~cm}$ of the chamber was constructed of horizontally mounted stainless steel rods, $5 \mathrm{~mm}$ in diameter and spaced $1.5 \mathrm{~cm}$ apart (center to center). The test chamber was located in a sound-attenuating chamber (IAC; Industrial Acoustics, New York). The tone CS was presented through a Jensen speaker (Model J1176) mounted in the ceiling of the IAC. A 7-W white light was mounted on an inner wall of the IAC to provide constant low-level illumination. Behavior during the test session was videotaped using a Sony video camera (Model CCD-TRV67), and the videotapes were later scored to measure levels of freezing behavior in the different groups.

Procedure. Animals of a given age were randomly assigned to one of four conditioning groups: short delay, long delay, trace, or unpaired ( $n$ s $=8-10 /$ group). The two delay groups differed in the duration of the CS that was paired with shock. For the short-delay group, the subjects were exposed to 10 presentations of a 10 -sec tone CS that terminated with the onset of the shock US. For the long-delay group, a 20-sec tone CS terminated with onset of the shock US. For the trace conditioning group, a 10-sec tone CS was 
presented and then separated from onset of the US by a 10 -sec stimulus-free trace interval. For the unpaired group, 10-sec CSs and USs were presented in an explicitly unpaired manner. USs were presented during each intertrial interval (ITI), with the stipulation that a US could not occur $60 \mathrm{sec}$ prior to or following a CS. For all the groups, ITIs ranged from 200 to $300 \mathrm{sec}$. The subjects were given a 10 -min period of adaptation to the conditioning context prior to the first stimulus presentation. The animals were removed $60 \mathrm{sec}$ after the final shock. The single training session lasted $1 \mathrm{~h}$.

Testing occurred approximately $24 \mathrm{~h}$ later in a novel context. The animals were placed into the test chamber and were exposed to a 10 -min period of adaptation. The subjects were then presented three nonreinforced 10 -sec tone CSs separated by 90 - to 120 -sec intervals, and the test sessions were videotaped. The videotapes were later scored for freezing by an observer blind to the conditioning group, using a time-sampling procedure (Fanselow, 1980). For $10 \mathrm{sec}$ prior to each stimulus and during the $10 \mathrm{sec}$ of the CS, the subject was briefly observed once every $2 \mathrm{sec}$ for the presence/absence of freezing. Freezing was defined as the absence of observable movements, except those necessary for respiration. The data were converted into a percentage of intervals scored as freezing (ranging from $0 \%$ to $100 \%)$.

\section{Results and Discussion}

An initial analysis conducted on the freezing data obtained from the three test trials yielded several interactions with the trial factor [smallest $F(6,262)=2.33, p<.01$ ]. These effects were due primarily to a substantial increase in pre-CS freezing on Trials 2 and 3. Therefore, only the data obtained from the first test trial will be reported.

An analysis of pre-CS freezing on the first test trial yielded no effects of age or conditioning treatment [largest $F(9,131)=1.73]$. The data were converted into a change score (CS freezing - pre-CS freezing) for analysis and presentation. Change scores for the different groups dur- ing the freezing test are shown in Figure 1. The data in Figure 1 reveal two primary findings. First, short-delay fear conditioning was acquired by PD 16, an age at which trace and long-delay conditioning were not yet evident (see Moye \& Rudy, 1987). Second, trace and long-delay fear conditioning emerged at approximately the same age (PD 18). A 4 (age) $\times 4$ (conditioning treatment) betweengroups ANOVA conducted on freezing change scores yielded significant main effects of age and conditioning treatment and an age $\times$ conditioning treatment interaction [smallest $F(9,131)=1.98, p<.05$ ]. Post hoc comparisons (Fisher test, $p=.05$; Keppel, 1982) were conducted to evaluate the source of the interaction, and the results can be summarized as follows. For the 14-day-olds, none of the experimental groups differed from the unpaired group. By PD 16, the short-delay group had significantly higher freezing scores than did the unpaired control, indicating the emergence of short-delay conditioning, which was maintained on PD 18 and PD 25. Beginning on PD 18, the trace and long-delay groups had significantly higher freezing scores than did the unpaired control, and on both PD 18 and PD 25, the trace and the long-delay groups did not differ.

Ivkovich et al. (2000) reported a similar pattern of development of delay and trace conditioning of the eyeblink response in immature rats. In Ivkovich et al., short-delay eyeblink conditioning was observed by PD 24 , whereas trace and long-delay conditioned responses were not as strongly evident until approximately PD 30 . These findings, combined with the present observation that trace and long-delay fear conditioning in the rat have a similar emergence, are important. They suggest the possibility

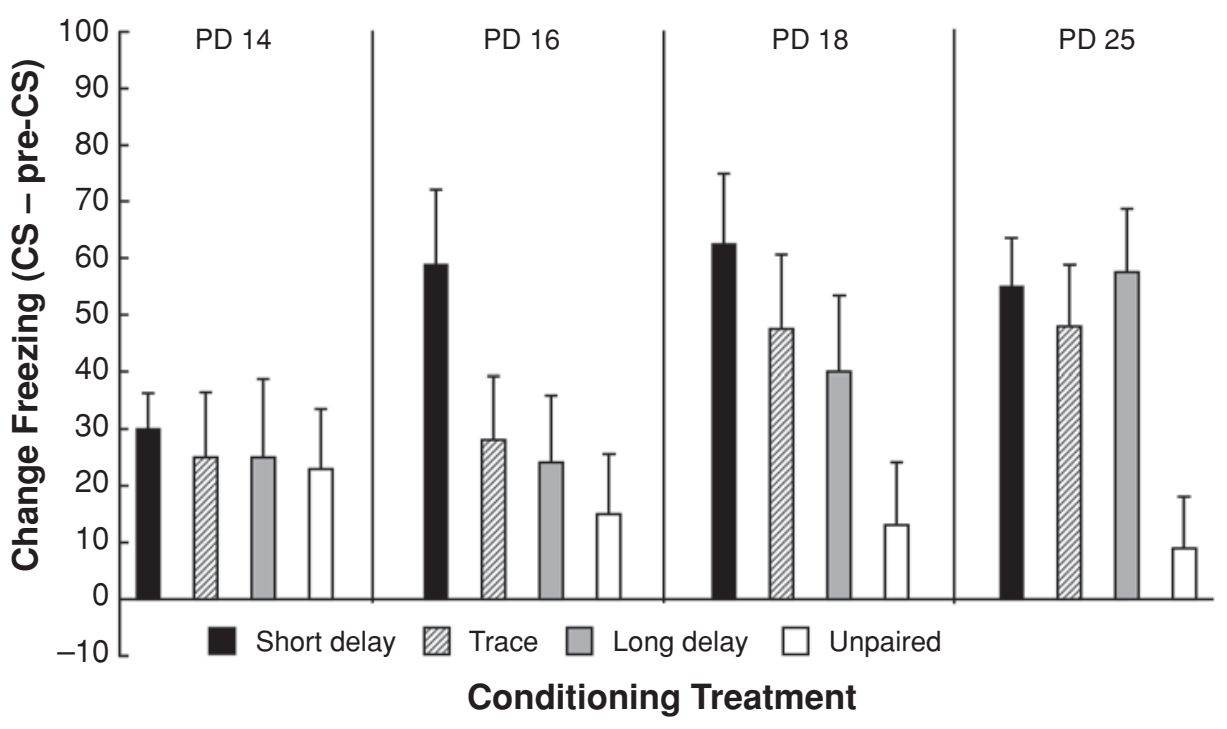

Figure 1. Mean $( \pm S E M)$ change in freezing $(\% \mathrm{CS}$ freezing $-\%$ pre-CS freezing) to an auditory stimulus recorded $24 \mathrm{~h}$ after training in Experiment 1. The animals ranged in age from 14 to 25 days old and were assigned to one of four conditioning groups: short delay, 10-sec CS immediately followed by shock; trace, 10-sec CS followed by 10-sec trace interval followed by shock; long delay, 20-sec CS immediately followed by shock; unpaired, explicitly unpaired CS and US presentations. PD, postnatal day. 
that the late emergence of trace conditioning observed here and by Ivkovich et al. may reflect the longer ISI used during training, rather than the imposition of an unfilled gap per se.

\section{EXPERIMENT 2}

Moye and Rudy (1987) have reported evidence suggesting that the capacity to learn about a visual CS in both delay and trace procedures does not emerge until a few days after the capacity to learn about an auditory CS (see also Hunt \& Campbell, 1997). The purpose of Experiment 2 was to assess whether the pattern of outcomes observed in Experiment 1 would be observed with a visual, as opposed to an auditory, CS during development of trace, short-delay, and long-delay conditioning. To the extent that similar patterns across delay and trace conditioning are observed, this would suggest that the ontogenesis of sensory information processing in different modalities follows developmentally locked, but parallel, patterns of emergence. Of central interest was whether the developmental pattern of trace and long-delay conditioning would also be in parallel. In Experiment 2, conditioned responding following short-delay, long-delay, and trace procedures was compared. The design of the experiment was the same as that in Experiment 1, except for modality of the CS.

\section{Method}

Subjects. The subjects were 173 male and female rats representing 22 different litters. On the day of training, the subjects were 18, $21,25,28$, or 32 days of age. One male and 1 female pup per litter were assigned to each of the four conditioning treatments within an age group ( $n \mathrm{~s}=8-10 /$ group).

Apparatus and Procedure. Training and testing occurred in the same apparatuses as those used in Experiment 1. The visual CS was produced by a $25-\mathrm{W}$ white bulb with its center located $12 \mathrm{~cm}$ above the floor and $8.5 \mathrm{~cm}$ from the rear of both the training and the testing chambers. The light flashed at a rate of $2 / \mathrm{sec}$. The subjects were randomly assigned to the short-delay, long-delay, trace, and unpaired groups, and all the conditions were identical to those in the previous experiment, except for the modality of the CS.

\section{Results and Discussion}

Levels of pre-CS freezing did not differ by age or conditioning treatment $[$ largest $F(4,153)=1.25]$. Freezing change scores (CS freezing - pre-CS freezing) are presented in Figure 2. As can be seen in Figure 2, short-delay conditioning was first evident at younger ages than were trace and long-delay conditioning (PD 18 vs. PD 25). In addition, trace and long-delay conditioning emerged at a similar age (between PD 21 and PD 25), and the magnitude of conditioned responding in trace and long-delay groups was similar at all ages. A 5 (age) $\times 4$ (conditioning treatment) ANOVA conducted on the freezing change scores obtained from the first test trial revealed significant main effects of age and conditioning treatment and an age $\times$ conditioning treatment interaction [smallest $F(4,153)=$ $2.70, p<.05]$. Post hoc comparisons (Fisher test) indicated significantly higher freezing in delay groups than in unpaired groups at all ages. Trace and long-delay performance did not differ from that of unpaired controls until PD 25, indicating the emergence of trace and long-delay conditioning. At this age, responding to the CS following trace or long-delay conditioning was less than that following short-delay conditioning. By 28 days of age, the magnitude of conditioned freezing did not differ among the short-delay, trace, and long-delay groups. Finally, at no age did trace and long-delay groups differ from one another in terms of the CS-elicited freezing observed.

Like the findings in Experiment 1, the results of Experiment 2 show the later development of trace, relative

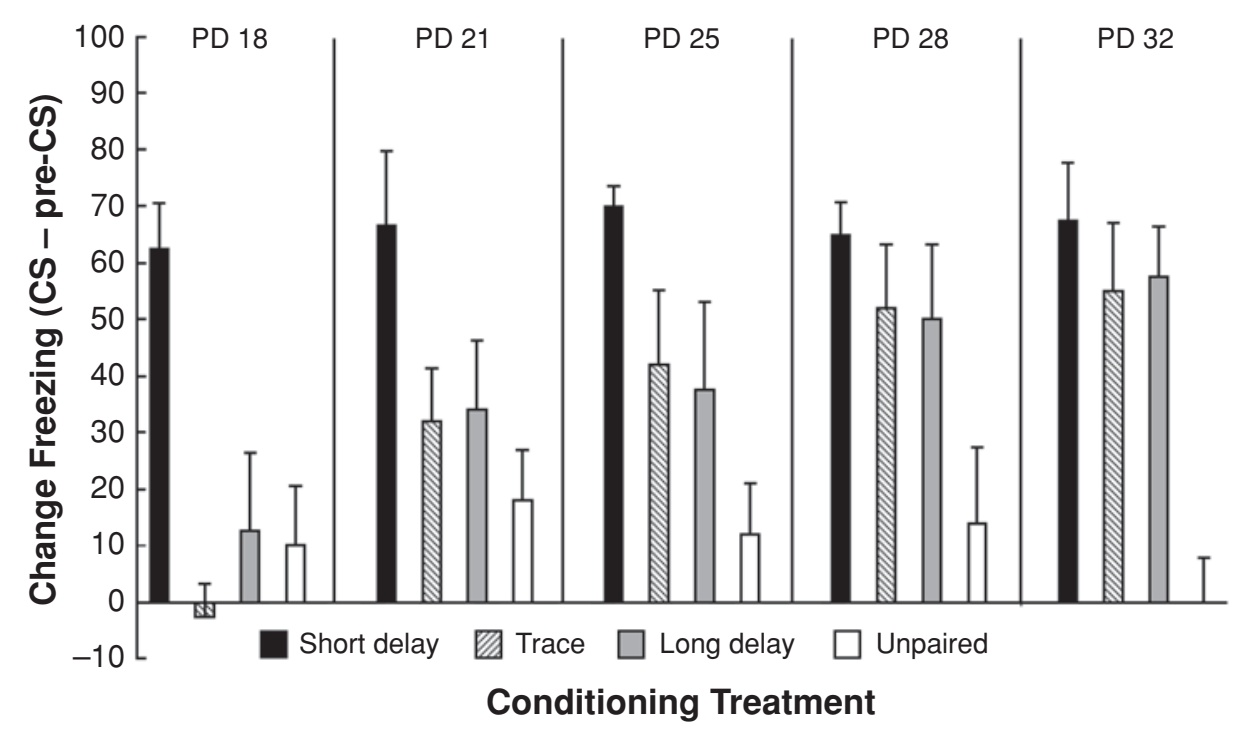

Figure 2. Mean $( \pm S E M)$ change in freezing to a visual CS in Experiment 2. The animals ranged in age from 18 to 32 days old. PD, postnatal day. 
to short-delay, fear conditioning. Of critical interest, they indicate the parallel emergence of trace and long-delay conditioning with a visual, as opposed to an auditory, CS. A summary of the ontogenetic emergence of trace and long-delay conditioning in both experiments is shown in Figure 3. Freezing data for the trace and long-delay conditioning groups at each age were transformed into a percentage of delay responding. This figure is intended to show that conditioned responding following trace and long-delay training procedures exhibit a remarkably parallel developmental trajectory. Across a range of ages and with two different modality CSs, the trace and long-delay groups show similar, if not identical, levels of conditioned responding.

\section{GENERAL DISCUSSION}

Collectively, the results of Experiments 1 and 2 show that trace and long-delay conditioning follow a parallel ontogenetic sequence. For both auditory and visual CSs, conditioned freezing following trace and long-delay training procedures were highly similar, and both were evident later in development than was short-delay conditioning. Previous studies of trace fear conditioning (e.g., Moye \& Rudy, 1987) have suggested that the later development of trace conditioning reflects the ontogeny of short-term memory processes. Young animals typically show inferior memory performance, as compared with older animals (e.g., Campbell \& Spear, 1972; Kail \& Spear, 1984), and the late ontogenetic emergence of trace conditioning is consistent with this general finding. The present results call into question the conclusion that it is solely the intervening trace interval that contributes to inferior conditioning with this procedure, at least in the developing rat. These findings are similar to those observed by Ivkovich et al. (2000), using the eyeblink conditioning preparation. However, the present experiments, as well as those of Ivkovich et al., employed only a single trace interval duration and long-delay ISI. It therefore remains possible that the ontogenetic profiles of trace and long-delay conditioning would diverge with a wider range of ISI durations.

The neural systems underlying the acquisition and expression of delay conditioned fear have been largely identified (e.g., Davis, 1992; LeDoux, 2000). It is also recognized that the hippocampus plays a critical role in trace conditioning (McEchron, Bouwmeester, Tseng, Weiss, \& Disterhoft, 1998; McEchron, Tseng, \& Disterhoft, 2000; Quinn, Oommen, Morrison, \& Fanselow, 2002; Solomon, Vander Schaaf, Thompson, \& Weisz, 1986). It has been hypothesized that a hippocampal-dependent short-term memory system is necessary to create and maintain a representation of the CS during the trace interval, allowing for the integration of the temporally discontiguous CS and US events (Rodriguez \& Levy, 2001; Wallenstein, Eichenbaum, \& Hasselmo, 1998). If the late ontogenetic emergence of trace conditioning is due to hippocampal immaturity, as has previously been suggested (Ivkovich \& Stanton, 2001; Moye \& Rudy, 1987), and delay conditioning is not hippocampal dependent, it remains unanswered why long-delay conditioning would also be developmentally delayed (like trace conditioning) and, furthermore, why long-delay and trace conditioning would emerge at the same time.

One possibility is that neural systems outside of the hippocampus mediate long-delay learning but that these systems develop in tandem with the hippocampus. Some studies have been performed to specifically examine a possible role for the hippocampus in long-delay conditioning, and the results have confirmed that hippocampal lesions selectively impact trace, but not long-delay,

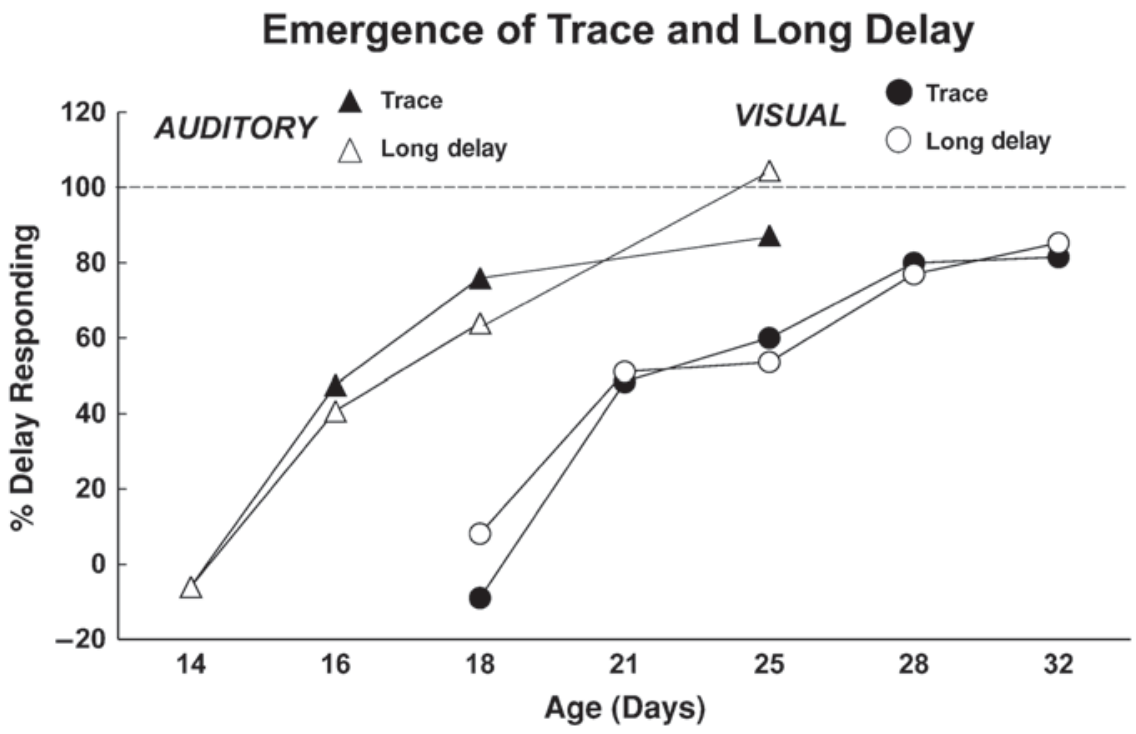

Figure 3. Summary of the findings in Experiments 1 (auditory CS) and 2 (visual CS) showing the parallel development of trace and long-delay conditioned responding. The data from these groups were converted into a percentage of short-delay responding for presentation. 
conditioning, at least with eyeblink preparations (Beylin, Gandhi, Wood, Talk, Matzel, \& Shors, 2001; Ivkovich \& Stanton, 2001). Interestingly, however, Beylin et al. did report that lesions of the hippocampus had detrimental effects on acquisition of the eyeblink response in a delay group that was trained with a "very long" duration CS. This group was included to match performance levels in trace and delay conditioning groups. Beylin et al. suggested that the hippocampus may be required when the task is made sufficiently difficult, as can be inferred from the similar rates of acquisition for trace and long-delay groups. In the present experiments, long-delay and trace groups at all ages exhibited comparable levels of performance, which might suggest that the long-delay procedure was particularly difficult for the young animals to learn. If so, it is not unreasonable to posit hippocampal involvement in the long-delay groups, at least with young animals (but see Ivkovich \& Stanton, 2001). The present experiments were not intended to directly address the putative role of the hippocampus in trace or long-delay conditioning, but they are relevant to a continuing assessment of what role the hippocampus plays in integrating memories for CSs and USs in classical conditioning. In particular, the results suggest that behavioral deficits in trace conditioning may be mediated, in part, by a long ISI, as opposed to the unfilled stimulus-free interval, or gap, in the trace procedure that is most commonly thought to be a critical feature of the trace conditioning deficit.

Other evidence invites reconsideration of what role the unfilled gap plays in the trace conditioning deficit. Cole, Barnet, and Miller (1995) have argued that the typically observed behavioral inferiority of trace, relative to shortdelay, conditioning reflects the expression of different temporal information encoded in the associations between CSs and USs, rather than inferior learning per se after trace conditioning (see also Burman \& Gewirtz, 2004; Kehoe \& Weidemann, 1999). Specifically, Cole et al. suggested that rats learn ISIs between CSs and USs and that the learning of a long ISI (as in trace conditioning) is expressed as a behavioral deficit, and they provided evidence of substantial learning following trace conditioning when measured indirectly with second-order and sensory preconditioning. Their results are generally consistent with the present experiments in that, when ISI was equated across the trace and long-delay conditions in Experiments 1 and 2, similar levels of conditioning were observed. Such an outcome would be anticipated if learning about ISIs between CSs and USs is a critical determinant of how learned associations are expressed in behavior.

The majority of behavioral and neurobiological studies of trace conditioning have employed delay conditioning controls that have been equated for CS duration and, thus, have confounded ISI. The present results, as well as those of Ivkovich et al. (2000), argue for the inclusion of a longdelay group in studies of trace conditioning, because such a group allows for evaluation of the roles of ISI and trace interval. Comparisons between trace and long-delay conditions are important because they contribute to assessing the functional characteristics of neural systems that serve short-term memory. Specifically, these comparisons invite analysis of whether the neural systems responsible for bridging the unfilled gap in trace conditioning are similar to, or independent from, the neural systems responsible for bridging the usually longer temporal delay from CS onset to US onset that occurs in long-delay conditioning. Concerning development, an already substantial literature indicates that some memory systems, including those that are hippocampal based, exhibit a late ontogenetic emergence (for a review, see Stanton, 2000). Developmentally delayed or impaired conditioning in tasks that require memory integration over a long delay and that may be mediated by systems outside of the hippocampus remains to be studied.

\section{REFERENCES}

Alberts, J. R. (1984). Sensory-perceptual development in the Norway rat: A view toward comparative studies. In R. Kail \& N. E. Spear (Eds.), Comparative perspectives on the development of memory (pp. 65-101). Hillsdale, NJ: Erlbaum.

Beylin, A. V., Gandhi, C. C., Wood, G. E., Talk, A. C., Matzel, L. D., \& Shors, T. J. (2001). The role of the hippocampus in trace conditioning: Temporal discontinuity or task difficulty? Neurobiology of Learning \& Memory, 76, 447-461.

Burman, M. A., \& Gewirtz, J. C. (2004). Timing of fear expression in trace and delay conditioning measured by fear-potentiated startle in rats. Learning \& Memory, 11, 205-212.

CAmpbell, B. A., \& Ampuero, M. X. (1985). Dissociation of autonomic and behavioral components of conditioned fear during development in the rat. Behavioral Neuroscience, 99, 1089-1102.

Campbell, B. A., \& Spear, N. E. (1972). Ontogeny of memory. Psychological Review, 79, 215-236.

Cole, R. P., Barnet, R. C., \& Miller, R. R. (1995). Temporal encoding in trace conditioning. Animal Learning \& Behavior, 23, 144-153.

Davis, M. (1992). The role of the amygdala in fear and anxiety. Annual Review of Neuroscience, 15, 353-375.

ELlison, G. D. (1964). Differential salivary conditioning to traces. Journal of Comparative \& Physiological Psychology, 57, 373-380.

FANSELOW, M. S. (1980). Conditional and unconditional components of post-shock freezing. Pavlovian Journal of Biological Sciences, 15, 177-182.

Hunt, P. S., \& CAmpbell, B. A. (1997). Developmental dissociation of the components of conditioned fear. In M. E. Bouton \& M. S. Fanselow (Eds.), Learning, motivation and cognition: The functional behaviorism of Robert C. Bolles (pp. 53-74). Washington, DC: American Psychological Association.

Ivkovich, D., PaczKowski, C. M., \& Stanton, M. E. (2000). Ontogeny of delay versus trace eyeblink conditioning in the rat. Developmental Psychobiology, 36, 148-160.

Ivkovich, D., \& Stanton, M. E. (2001). Effects of early hippocampal lesions on trace, delay, and long-delay eyeblink conditioning in developing rats. Neurobiology of Learning \& Memory, 76, 426-446.

KaIL, R., \& SpEAR, N. E. (EDS.) (1984). Comparative perspectives on the development of memory. Hillsdale, $\mathrm{NJ}$ : Erlbaum.

KAMIN, L. J. (1954). Traumatic avoidance learning: The effects of CSUS interval with a trace conditioning procedure. Journal of Comparative \& Physiological Psychology, 47, 65-72.

Kehoe, E. J., \& WeidemanN, G. (1999). Within-stimulus competition in trace conditioning of the rabbit's nictitating membrane response. Psychobiology, 27, 72-84.

KEPPEL, G. (1982). Design and analysis: A researcher's handbook. Englewood Cliffs, NJ: Prentice-Hall.

LeDoux, J. E. (2000). Emotion circuits in the brain. Annual Review of Neuroscience, 23, 155-184.

McEchron, M. D., Bouwmeester, H., Tseng, W., Weiss, C., \& DisterhofT, J. F. (1998). Hippocampectomy disrupts auditory trace fear conditioning and contextual fear conditioning in the rat. Hippocampus, 8, 638-646. 
McEchron, M. D., Tseng, W., \& Disterhoft, J. F. (2000). Neurotoxic lesions of the dorsal hippocampus disrupt auditory-cued trace heart rate (fear) conditioning in rabbits. Hippocampus, 10, 739-751.

Moye, T. B., \& Rudy, J. W. (1987). Ontogenesis of trace conditioning in young rats: Dissociation of associative and memory processes. Developmental Psychobiology, 20, 405-414.

Quinn, J. J., Oommen, S. S., Morrison, G. E., \& Fanselow, M. S. (2002). Post-training excitotoxic lesions of the dorsal hippocampus attenuate forward trace, backward trace, and delay fear conditioning in a temporally specific manner. Hippocampus, 12, 495-504.

Rodriguez, P., \& LEVY, W. B. (2001). A model of hippocampal activity in trace conditioning: Where's the trace? Behavioral Neuroscience, 115, 1224-1238.
Solomon, P. R., Vander SchaAf, E. R., Thompson, R. F., \& Weisz, D. J. (1986). Hippocampus and trace conditioning of the rabbit's classically conditioned nictitating membrane response. Behavioral Neuroscience, 100, 729-744.

Stanton, M. E. (2000). Multiple memory systems, development and conditioning. Behavioural Brain Research, 110, 25-37.

Wallenstein, G., Eichenbaum, H., \& Hasselmo, M. (1998). The hippocampus as an associator of discontiguous events. Trends in Neurosciences, 21, 317-323.

(Manuscript received November 3, 2004; revision accepted for publication March 23, 2005.) 